\title{
Sensitivity Analysis on Temperature Influence Factors of Refrigerated
}

\author{
Truck \\ Jin $\mathrm{Li}^{1, \mathrm{a}^{*}}$ and Chang-zheng Huang ${ }^{1, \mathrm{~b}}$ \\ ${ }^{1}$ School of Physics and Mechanical \& Electrical Engineering, Shaoguan University, Shaoguan, \\ China, 512005
}

\section{a653251849@qq.com, bhczchn@163.com}

Keywords: Influencing parameters; sensitive factors; sensitive weights; compartment temperatures Abstract: In order to get the influence parameters on the temperature in the refrigerated compartment, the influence of temperature on the temperature of the refrigerated compartment was established, and the influence of the sensitive factors on the temperature of the refrigerated compartment was analyzed. The results show that the temperature inside refrigerated truck is the most sensitive to the insulation materials thermal conductivity of refrigerated compartments, the insulating material thickness of refrigerated compartments and the leakage ratio of the refrigerated compartment. When insulating material thickness of refrigerated compartment in the range of $0 \sim$ $0.2 \mathrm{~m}$, sensitive weights decreased exponentially from 16 to 1.8 . When the insulating material thermal conductivity of refrigerated compartment increases at $0 \sim 0.045 \mathrm{w} /(\mathrm{m} \cdot \mathrm{k})$, sensitive weights decreased inversely from 7.8 to 7.15 in proportion. And when the leakage ratio of refrigerated compartments changes in the range of $0 \sim 1$, sensitive weights exponentially decreased from 3 to 0.2. Moreover, other influencing parameters have little influence on the temperature of refrigerated compartments.

\section{Introduction}

The accurate control of the temperature inside refrigerated trucks is one of the key techniques to study and design the refrigerated truck, as well as the key way to guarantee the quality of the goods for transport in the compartment. So, it's key to find main factors which affect the accurate control of the temperature inside refrigerated trucks ${ }^{[1 \sim 4]}$. Thus, Li Jin et al. found the main causes ${ }^{[1 \sim 6]}$ by establishing cooling mathematical model, analyzing influencing factors on the cooling rate, cooling time, as well as the non-uniformity of temperature fluctuations and temperature distribution inside refrigerated trucks. Long Enshen, Liu Hongsheng et al.. also have studied temperature characteristics and influence factors in the passenger cars' compartments, by testing and numerical modeling ${ }^{[7 \sim 10]}$ etc. Virseda, Sesttrada et al. have analyzed the main factors causing the compartment or refrigerator compartment temperature fluctuations by using Laplace transform and transfer function $^{[11 \sim 13]}$. The studies above have a certain significance to control the inside temperature fluctuations, to improve the cooling rate, cooling time of refrigerated trucks. But none of them did establish a quantitative model to analyze the impact of various factors within the refrigeration compartment temperature control accuracy. For this reason, combined with the reality, the paper cited the sensitivity analysis method of the main factors affecting the temperature inside the refrigerated compartment of quantitative analysis, provide important theoretical basis for the refrigerated compartment design. 


\section{The temperature mathematical model of refrigerated truck}

\section{Modeling assumptions before}

Modeling analysis assumed refrigerated trucks parked long enough; Temperature inside refrigerated compartment is uniform throughout and changes over time; in the state of driving, the vehicle maintains uniform speed; The compartment can be viewed as a one-dimensional heat transfer; The compartment is interconnected by superposition of multiple layers of material in the thickness direction; the same compartment surface is of the same physical properties; Refrigerated trucks in the refrigeration cooling process are without any form of operations management.)

\section{Mathematical modeling}

According to the structure of refrigerated trucks and internal and external heat loads, the heat balance equation of refrigerated trucks can be established ${ }^{[1]}$

$$
\begin{aligned}
& c_{p} \rho V \frac{d t}{d \tau}=Q_{1}+Q_{2}+Q_{3}+Q_{4}+Q_{5}-Q_{0} \\
& Q_{1}=\sum\left(\frac{1}{\frac{1}{a_{w}}+\sum \frac{\delta_{i}}{\lambda_{i}}+\frac{1}{a_{i n}}}\right)\left(t_{i w}-t\right) F_{i} \\
& F_{i}=\sqrt{\left(L_{i w} \times W_{i w}\right)\left(L_{i n} \times W_{i n}\right)} \\
& Q_{2}=\frac{\beta \rho V}{3600}\left(c_{p}\left(t_{k}-t\right)+\gamma\left(\phi_{w} \chi_{w}-\phi_{i n} \chi_{i n}\right)\right) \\
& Q_{3}=\frac{1}{24 \times 3600} G H \\
& Q_{5}=\mathrm{N} \zeta \zeta \\
& a_{w}=1.163 \times\left(4+12 \sqrt{v_{c}}\right) \\
& a_{i n}=1.163 \times\left(4+12 \sqrt{v_{k}}\right)
\end{aligned}
$$

Where: $a_{w^{-}}$-compartments in vitro surface convective heat transfer coefficient, $\mathrm{W} / \mathrm{m}^{2} ; a_{i n}$-compartments body surface heat transfer coefficient, $\mathrm{W} / \mathrm{m}^{2} ; \delta_{i^{--}}$thickness of the heat exchange surface, $\mathrm{m} ; \lambda_{i^{--}}$each layer on the surface heat transfer coefficient of thermal conductivity, W / $(\mathrm{m}$ $\left.{ }^{\circ} \mathrm{C}\right) ; t_{i w^{--}}$compartments in vitro surface convert temperature, ${ }^{\circ} \mathrm{C} ; t$-- flowing air temperature in the compartment, ${ }^{\circ} \mathrm{C}$; $t_{k^{--}}$sometime in a place outside the compartment air temperature, ${ }^{\circ} \mathrm{C}$; $\tau$-- time, s; $F_{i^{--}}$compartment body heat transfer area, $m^{2} ; L_{i w^{-}}$-the length of the compartment body surface, $\mathrm{m}$; $W_{i w^{--}}$the width of the compartment body surface, $\mathrm{m} ; L_{i n^{-}}-$the length of the compartment body surface, $\mathrm{m} ; W_{i n}$ - width or height of compartment body surface, $\mathrm{m} ; \beta$ - - refrigerated trucks of air leakage in multiples; $\gamma$-- the heat of condensation of water vapor cooled, $\mathrm{J} / \mathrm{kg} ; \phi_{w}, \phi_{i n}{ }^{-}-$trains respectively, relative humidity of the air inside; $x_{w}, x_{i n^{--}}$under the state of saturated air the moisture content of the outside and inside compartment, g / kg; $G$-- compartment cargo weight, $\mathrm{t}$; $H_{--}$ breathing hot vehicle cargo, $\mathrm{J} /(\mathrm{t} \cdot 24 \mathrm{~h}) ; N$ - - motor rated power, W; $\varepsilon$ - - heat transfer coefficient (full motor in the car, take $\varepsilon=1$ ); $\zeta$-- motor running time factor (due to the cooling process continuous motor work, take $\zeta=1) ; v_{c}{ }^{-}$- refrigerated trucks speed, $\mathrm{m} / \mathrm{s} ; v_{k}{ }^{-}$- inside air velocity, $\mathrm{m} / \mathrm{s}$.

Normally, the compartment is without any operation during refrigerated transport process, thus 
provided (1) where $\mathrm{Q}_{4}=0$, the simultaneous (1) (6), so that:

$$
\begin{aligned}
& A=-\left(\sum\left(\frac{1}{\frac{1}{a_{w}}+\sum \frac{\delta_{i}}{\lambda_{i}}+\frac{1}{a_{i n}}}\right)\left(\frac{\sqrt{\left(L_{i w} W_{i w}\right)\left(L_{i n} W_{i n}\right)}}{\rho V c_{p}}\right)+\frac{\beta}{3600}\right) \\
& B=\sum\left(\frac{1}{\left.\frac{1}{a_{w}}+\sum \frac{\delta_{i}}{\lambda_{i}}+\frac{1}{a_{i n}}\right)\left(\frac{\sqrt{\left(L_{i w} W_{i w}\right)\left(L_{i n} W_{i n}\right)}}{\rho V c_{p}}\right) t_{i w}+\frac{\beta t_{k}}{3600}}\right. \\
& +\frac{\beta \gamma\left(\phi_{w} x_{w}-\phi_{i n} x_{i n}\right)}{3600 c_{p}}+\frac{G H}{24 \times 3600 \rho V c_{p}}-\frac{Q_{o}}{\rho V c_{p}}
\end{aligned}
$$

So: $\quad \frac{d t}{d \tau}=A t+B$

formula (9) integral algorithm (when $\tau=0$ and the inside air temperature is $\mathrm{t}=\mathrm{t} 0$ ), so

$$
t=\left(t_{0}+\frac{B}{A}\right) e^{A \tau}-\frac{B}{A}
$$

\section{Compartment Surface Overall Temperature's Determination}

Compartments surface overall temperature is a surface temperature, synthesized from the the sun radiation, sky scattering radiation, ground reflected radiation, and air temperature ${ }^{[1]}$, namely:

$$
t_{i w}=t_{k}+\frac{p I}{a_{w}}-\frac{\varsigma \Delta R}{a_{w}}
$$

Where $p$-- absorption coefficient, compartments around the wall and the top surface (white outer surface) of fiberglass, take $\mathrm{p}=0.2$; I-- solar radiation intensity, $\mathrm{W} / \mathrm{m}^{2}$; Long-wave radiation $\zeta$-- body outer wall; $\Delta \mathrm{R}$-- long-wave radiation exchange volume car body surface between the sky and the surrounding objects.

When calculating, for vertical $\frac{\varsigma \Delta R}{a_{W}}$ take $0{ }^{\mathrm{o}} \mathrm{C}$, for horizontal $\frac{\varsigma \Delta R}{a_{W}}$ take $3.5 \sim 4{ }^{\mathrm{o}} \mathrm{C}$, this value is generally determined by the experience; For solar radiation intensity I, reference [1] to determine, not repeat them here.

\section{Experimental Verification}

Refrigerator trucks test was conducted in Guangzhou University refrigerated transport simulation test platform, the test platform structure is as shown in figure 1, and the details are as follows:

(1) Refrigerated trucks in the external environment simulation: To simulate the temperature changes over time outside refrigerated trucks, wrap a large body outside the refrigerated transport unit to simulate air temperature changes arbitrarily from $-20{ }^{\circ} \mathrm{C} \sim 50{ }^{\circ} \mathrm{C}$ outside the truck. With different vehicle speeds, set up six sets of large air handling units in front of the refrigerated transport unit, making the wind speed freely varied between $0 \sim 30 \mathrm{~m} / \mathrm{s}$ by frequency control, it can also simulate the compartment running at the speed of $0 \sim 120 \mathrm{~km} / \mathrm{h}$.

(2) Refrigerated transport compartment refrigerating and air supplying system Simulation: Refrigerated transport unit internal dimensions (length $\times$ width $\times$ height) are $4958 \mathrm{~mm} \times 2438 \mathrm{~mm}$ $\times 2590 \mathrm{~mm}$, internal unit body comprises of $3 \mathrm{~mm}$ fiberglass inside and $100 \mathrm{~mm}$ polyurethane insulation material outside. Equipped with corresponding heating, cooling systems and humidification devices, temperature inside the refrigerator unit can be freely adjusted between -25 $\sim 20{ }^{\circ} \mathrm{C}$, and relative humidity adjustable is in range of 0 to $100 \%$, and the unit (4NCS-12.2-type compressor) cooling capacity can be grade adjusted. This experiment is 3190W. Air supplying speed could adjust air volume in $0 \sim 2 \mathrm{~m} / \mathrm{s}$ by variable frequency fan, combined with the selection of 
the outlet opening size to achieve the goal of adjusting wind speed.

(3) Test Station Monitoring System: multiple temperature and humidity sensors were installed inside and outside the test station, so temperature and humidity parameters changes can be obtained timely. 50 fixed and movable temperature and humidity sensors were fixed up in the external environment simulation unit and internal refrigeration unit. In the front, middle and behind of the internal unit, 9 temperature and humidity sensors directly connected to computer were fixed up, which can collect data once per second. Winds inside and outside the compartment were measured with testo405-VI handheld wind speed instrument, and refrigerated transport unit outer surface use SATIR HM-200 infrared camera to measure the temperature. The above -mentioned parameters' average value were adopted as the final value.

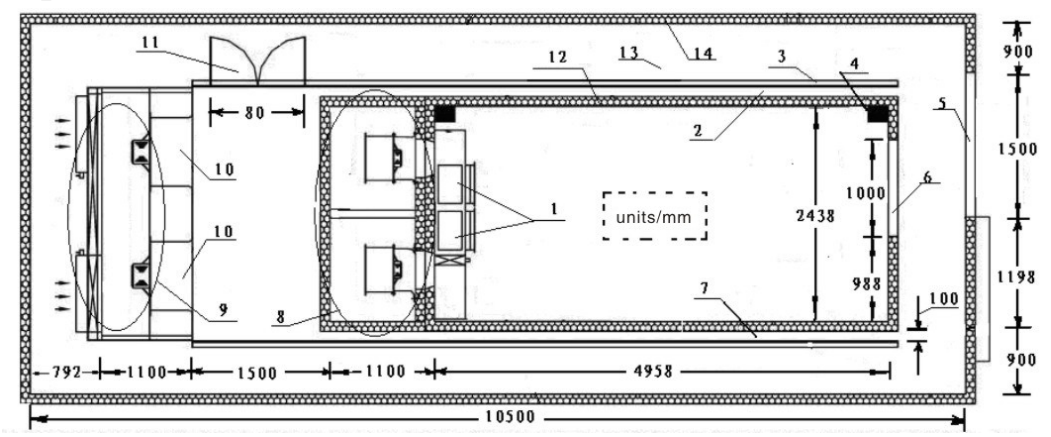

1. Evaporator 2. Environmental Simulation outside air supply duct 3.Separator 4.The temperature and humidity sensor junction box 5.Outside the door environment gate 6.Door internal environment 7. Environmental Simulation outside air supply duct 8.Internal Refrigerated Transport Unit 9.The external environment simulation assembly 10.The external environment control assembly outlet 11.Overhaul with side door 12.Overhaul with the environment outside insulation envelope 13.Return air duct outside environment 14.Thermal insulation enclosure structure of external environment

Fig.1 Simulation test platform of refrigerated transport technology

(4) Test results and analysis: Before cooling, Ambient temperature inside and outside compartment are set to $35{ }^{\circ} \mathrm{C}$ (keeping steady state for four hours), relative humidity (60 \pm 5$) \%$, termination temperature is $0{ }^{\circ} \mathrm{C}$, relative humidity $(40 \pm 5) \%$, evaporator air supply speed $4 \mathrm{~m} / \mathrm{s}$, test data is recorded once every minute, finally refrigerated trucks and theoretical temperature curve can be shown in Figure 2.

From Figure 2(a), 2(b), it can be seen that the theoretical calculation and the fileld measured cooling curve are basically the same, although when temperature suddenly drops to almost $0^{\circ} \mathrm{C}$, the test vehicle experienced a warming process at $40 \mathrm{~km} / \mathrm{h}$ with due to defrosting. Driving at $40 \mathrm{~km}$ / h, the relative error between the theoretically calculated and field measured curves before warming was $15 \%$. Driving at $80 \mathrm{~km} / \mathrm{h}$ the relative error between the theoretically calculated and field measured curves was $13 \%$, thus demonstrating the correctness of the mathematical model previously built. 


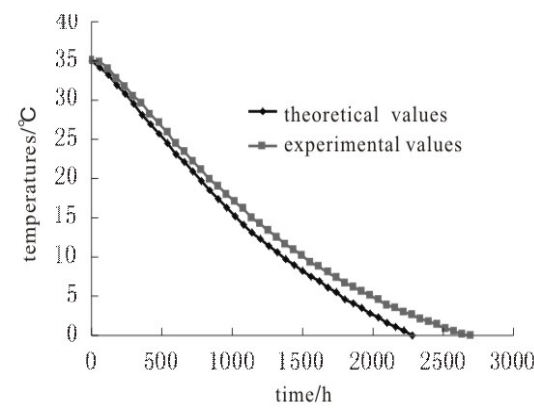

(a) $40 \mathrm{~km} / \mathrm{h}$

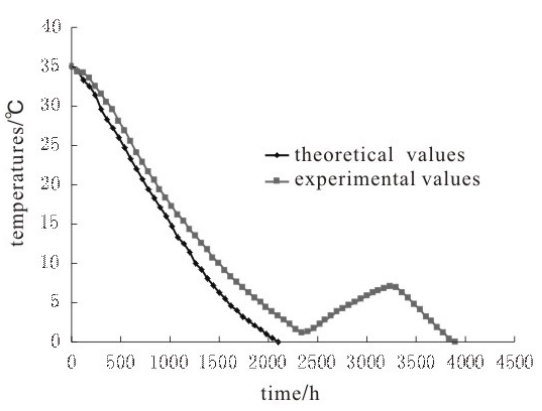

(b) $80 \mathrm{~km} / \mathrm{h}$

Fig.2 Refrigerated trucks' measured and theoretical temperature curve

\section{Sensitivity model}

\section{The definition of sensitive factor}

As can be seen from formula (10), refrigerated compartment temperature change model is non-linear model, therefore, refrigerated trucks at a certain state temperature parameters $\left(\lambda_{i} 、 \delta_{i}\right.$ 、 $\left.v_{c}, \beta, v_{k}, Q_{0}, p, H\right)$ can be described as non-linear equation)

$$
t=f\left(\lambda_{i}, \delta_{i}, \ldots, p, H\right)=f\left(\chi_{1}, \chi_{2}, \ldots \chi_{n}\right)
$$

Sensitivity analysis is a method for uncertainty analysis, by the formula (12), when all the factors change, respectively, by, $\chi_{1}, \chi_{2}, \ldots \chi_{n}$ become, $\chi_{1}^{\prime}, \chi_{2}^{\prime}, \ldots \chi_{n}^{\prime}$, variable $\Delta \chi_{1}, \Delta \chi_{2}, \cdots \Delta \chi_{i}, \ldots \Delta \chi_{n}$, the temperature in refrigerated trucks in the inevitable change, inside air temperature $t$ also changes into the $t^{\prime}$, can be represented by $\Delta t=t^{\prime}-t$ common cause of all the factors that change $\mathrm{t}$ the amount of change ${ }^{[14]}$. Using multivariate Taylor expansion function was:

$$
\Delta t \approx \frac{\partial t}{\partial x_{1}} \Delta x_{1} \cdots+\frac{\partial \mathrm{t}}{\partial x_{i}} \Delta x_{i} \cdots+\frac{\partial \mathrm{t}}{\partial x_{n}} \Delta x_{n}
$$

Refrigerated compartment temperature change by the analysis model shows that by some parameters deviation occurs is not objective changes, other parameters will not cause a change. Therefore, only the parameter $\chi_{i}$ changes, other parameters will not change. When the amount of $\mathrm{t}$ is $\Delta t_{x i}$, the impact on $\mathrm{t}$ can be expressed as:

$$
\Delta t_{x i}=\frac{\partial t}{\partial x_{i}} \Delta x_{i}
$$

The overall change of temperature refrigerated truck:

$$
\Delta t=\sum_{i=1}^{n} \Delta t_{x i}
$$

Define $\mathrm{t} / \chi_{i}$ as sensitive factor $\mathrm{B}$ of $\mathrm{t}$ to $\mathrm{A}$, there are:

$$
\mathrm{S}_{i}=\frac{\Delta t_{x i} / t}{\Delta x_{i} / x_{i}}=\frac{\frac{\partial t}{\partial x_{i}} \Delta x_{i} / t}{\Delta x_{i} / x_{i}}=\frac{\partial t}{\partial x_{i}} \cdot \frac{x_{i}}{t}
$$

$S_{i}$ is the certain parameters sensitivity of air temperature in the compartment. When it is a 
positive number, then the same direction change in $\Delta t_{x i}$ and $\Delta x_{i}$, whereas the opposite direction of change. By the formula (16) can be seen, the sensitivity factor $S_{i}$ of the refrigerator temperature influence factors is the partial derivative of parameter $x_{i}$.

\section{Determination of sensitive weight}

Respectively, formula (10) $\lambda_{i} 、 \delta_{i}, v_{c}, \beta, v_{k}, Q_{0}, p, H$ partial derivative, we can get all parameters sensitive factor (taking into account the space where expression omitted). In order to analyze each of the temperature-sensitive factor on the impact of refrigerated trucks, and further processing of sensitive factors, the method of which is ${ }^{[15]}$.

$$
S=\sum_{i=1}^{n} S_{i}
$$

the weight of each sensitive factor can be expressed as:

$$
\mathrm{S}_{i}^{t}=S_{i} / S
$$

under various initial conditions, the sensitive factor weights can be expressed as:

$\mathrm{S}_{i, j}^{t}=S_{i, j} / S_{i}^{m}$

$\mathrm{S}_{i}^{m}$ is the sum of a sensitive to initial conditions, namely:

$$
\mathrm{S}_{i}^{\mathrm{m}}=\sum_{j=1}^{n} S_{i, j}
$$

\section{Sensitivity Analysis}

\section{Parameter setting}

Refrigerated compartments related parameters of a refrigerator compartments manufacturers in Guangzhou related parameters shall prevail, specific see table 1 . The value of $\gamma_{、} \phi_{w} 、 \phi_{\text {in }} 、 x_{w} 、 x_{i n}$ 、 $C_{p}, N, \zeta$ of the same as the reference [1].

Tab.1Refrigerated trucks related parameter values

\begin{tabular}{cc|cc}
\hline parameters & parameter values & parameters & parameter values \\
\hline$\lambda_{i}$ & $0.033 \mathrm{~W} / \mathrm{m}^{\circ}{ }^{\circ} \mathrm{C}$ & $H$ & $1070 \mathrm{~kJ} \cdot(\mathrm{t} \cdot 24 \mathrm{~h})^{-1}$ \\
$\delta_{i}$ & $0.1 \mathrm{~m}$ & $G$ & $3 \mathrm{t}$ \\
$v_{c}$ & $80 \mathrm{~km} / \mathrm{h}$ & $L_{\text {in }}$ & $4.958 \mathrm{~m}$ \\
$\beta$ & 0.6 & $W_{\text {in }}$ & $2.438 \mathrm{~m}$ \\
$v_{k}$ & $1.2 \mathrm{~m} / \mathrm{s}$ & $L_{\text {iw }}$ & $5.064 \mathrm{~m}$ \\
$Q_{0}$ & $965 \mathrm{~W}$ & $W_{\text {iw }}$ & $2.544 \mathrm{~m}$ \\
$P$ & 0.2 & $I$ & $756 \mathrm{w} / \mathrm{m}^{2}$ \\
\hline
\end{tabular}

According to the above parameters, the time constant as a variable, available each sensitive factor $\left(S_{H} 、 S_{\delta_{i}} 、 S_{\beta} 、 S_{\lambda_{i}} 、 S_{Q_{o}} 、 S_{v_{c}} 、 S_{v_{k}} 、 S_{p}\right)$ expression (taking into account the space, where expressions can be omitted).

\section{Sensitive weight determination and analysis}

According to the model, the time constant Fig. 2(b) values substituted into sensitive factor 
formula, refrigerated trucks for temperature sensitive factors and sensitivity weighting factor calculation, the results in Table 2 below.

\begin{tabular}{ccccc}
\multicolumn{5}{c}{ Tab.2 Sensitive factor and sensitive weight of temperature } \\
\hline parameters & $\lambda_{i}$ & $\delta_{i}$ & $\beta$ & $v_{c}$ \\
\hline Sensitive factors & $-1.38 \times 10^{8}$ & $4.54 \times 10^{7}$ & $7.70 \times 10^{6}$ & -48.27 \\
weights & 7.65 & 2.11 & 0.50 & $2.68 \times 10^{-4}$ \\
\hline parameters & $v_{k}$ & $Q_{0}$ & $P$ & $H$ \\
\hline sensitive factors & -2277.2 & -0.25 & 1.10 & $8.62 \times 10^{-6}$ \\
weights & $8.26 \times 10^{-3}$ & $1.39 \times 10^{-8}$ & $6.10 \times 10^{-8}$ & $4.82 \times 10^{-13}$ \\
\hline
\end{tabular}

Table 2 shows, refrigerated trucks sensitivity of each factor varies, within the refrigerator compartment temperature sensitive right major influencing factors were in the order $\delta_{i}, \lambda_{i} 、 \beta, v_{k}$ 、 $v_{c}, p 、 Q_{0}, H$, wherein the inside temperature is the most sensitive to $\delta_{i}, \lambda_{i}, \beta$.

Similarly, according to the related parameters in table 1 and the sensitivity factor, you can get the change of the parameters related to relevant parameters of the sensitive weight, as shown in figure 3.

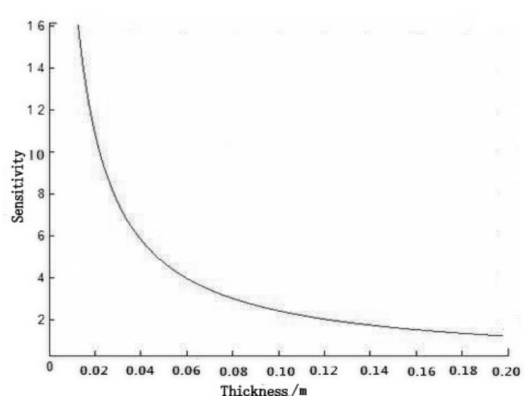

(a)

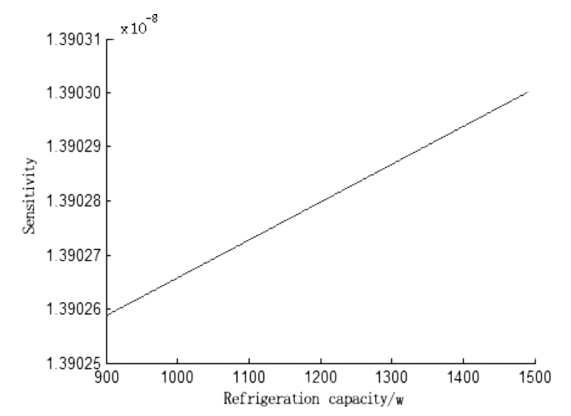

(c)

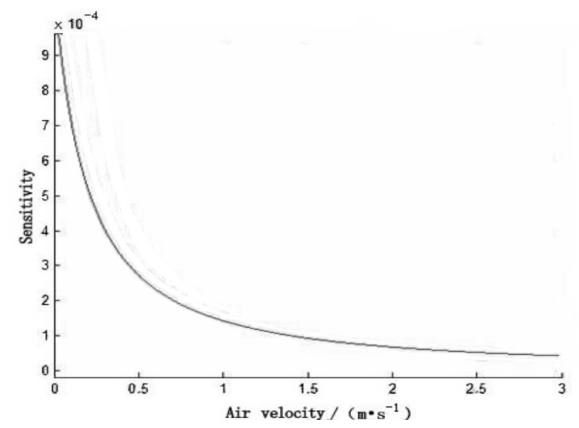

(e)

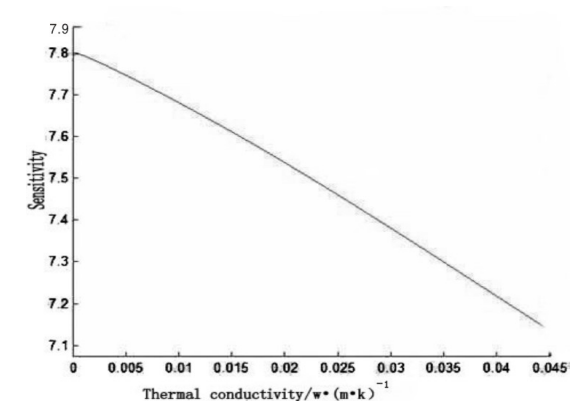

(b)

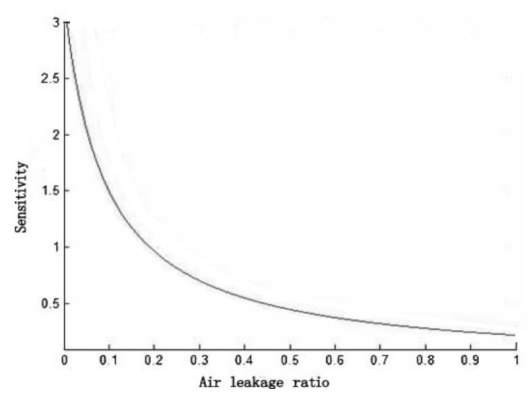

(d)

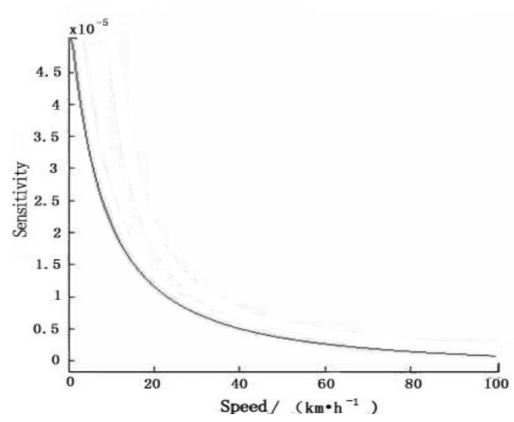

(f) 


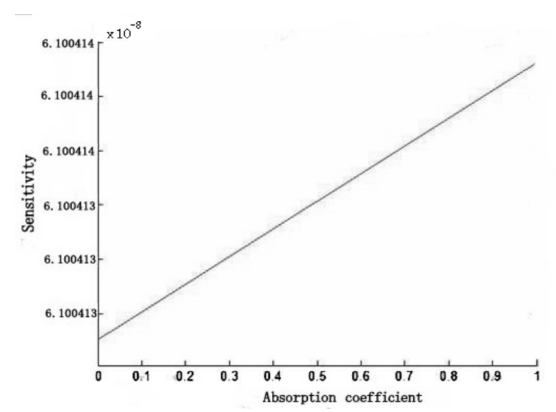

(g)

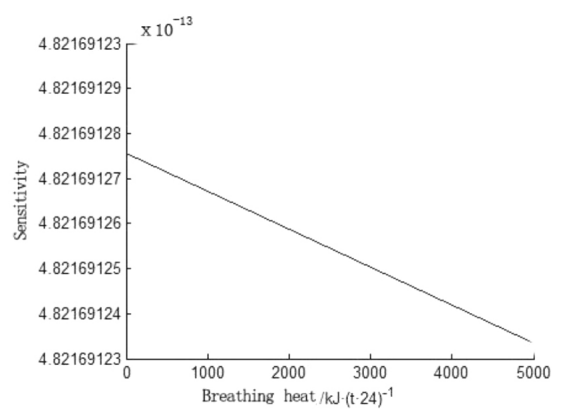

(h)

((a) changes in the thermal conductivity of the insulation material (b) changes in the thickness of the insulation material (c) leak fold change (d) changes in the cooling capacity (E)the vehicle speed change (f)air velocity variation (g) the cargo respiration thermal variations (h) the solar radiation absorption coefficient of variation)

Fig. 3 The sensitivity curves on a parameter

Figure 3 in Table 1 is the sensitivity weight curve; under the condition that other relevant parameters and each sensitivity factor remain the same while only one parameter changes. Figure 3(a) shows that when the thermal conductivity of insulation materials ranges from $0 \sim 0.045 \mathrm{w} \bullet(\mathrm{m} \bullet$ $\mathrm{k})^{-1}$, the sensitivity weight value is reduced from 7.8 to 7.15 , moreover, both form an inverse relationship; indicating that the thermal conductivity parameter changes of insulation material have great influence on the temperature inside the compartment. In Figure 3(b), when the compartment insulation materials' thickness ranges from $0.02 \sim 0.2 \mathrm{~m}$, the thickness of insulation material affect greatly the temperature inside the compartment, sensitive weight value decreased from 16 to 1.8 , and both decrease following the law of exponential, first fast and later slowly.

Figure 3(c) shows that when compartment leakage multiple changes from $0 \sim 1$, similar to the influence insulation material thickness variation on the compartment, both decrease following the law of exponential, first fast and later slowly, and sensitive weight value decreased from 3 to 0.2 . Figure 3(d) shows that when the cooling capacity $Q_{0}$ ranges from $900 \sim 1500 \mathrm{w}$, there are small changes in weight values in $10^{-8}$ level, indicating that the changes in the cooling capacity affect the weight weakly. Figure 3(e) and figure 3(f) show the sensitivity weight change chart when Vehicle $v_{c}$ is $0 \sim 100 \mathrm{~km} \cdot \mathrm{h}^{-1}$ and air velocity inside the compartment $v_{k}$ is $0 \sim 3 \mathrm{~m} \cdot \mathrm{s}^{-1}$ respectively. As can be seen from the diagram, sensitive weight value of $v_{c}$, the air velocity inside the compartment showed exponential increase tendency. Air velocity inside the compartment affects weight relatively greater, both were in $10^{-5}$ and $10^{-4}$ two levels respectively, and its effect on sensitivity weight absolute value is nearly minimal. As can be seen from figure $3(\mathrm{~g})$, refrigerated trucks produce different respiratory heat with different goods. When respiratory heat $\mathrm{H}$ is $0 \sim 5000 \mathrm{~kJ} \cdot(\mathrm{t} \cdot 24 \mathrm{~h})^{-1}$, the weight value shows subtle changes in direct proportion relationship within $10^{-13}$ level; Its effect on sensitive weight value is negligible. Figure $3(\mathrm{~h})$ is the sensitivity weight change chart, taking the outer surface of the compartment absorb solar radiation's coefficient $\mathrm{p}$ ranges from $0 \sim 1$, whose weight value is inversely proportional to the subtle changes occurring in the $10^{-8}$ level, the impact on the weight is also negligible.

\section{Conclusions}

Introducing sensitivity analysis to the refrigerated compartment temperature parameter analysis, the study found that refrigerated compartment temperature is the most sensitive to the thermal conductivity of insulation material $\lambda_{i}$, insulation thickness $\delta_{i}$ and multiple compartments leakage $\beta$ changes. When refrigerated compartment insulation material thickness becomes large in the range 
of $0 \sim 0.2 \mathrm{~m}$, sensitivity weight exponentially decreased from 16 to 1.8 . When refrigerated compartment insulation material thermal conductivity value increases at $0 \sim 0.045 \mathrm{w} \cdot(\mathrm{m} \cdot \mathrm{k})^{-1}$, the sensitive weights inversely law decreases from 7.8 to 7.15 , and when refrigerated trucks leakage multiple changes from $0 \sim 1$, sensitivity weight value decrease exponentially from 3 down to 0.2. Other influence parameters on the temperature inside the refrigerator compartment have little effect.

\section{Acknowledgements}

This work was financially supported by the National scientific and technological plan (No.2013BAD9B01-1) and Guangdong natural science foundation (No.2015A 030310411) and Guangdong province higher education outstanding young teachers training program (No.YQ 2014156) and Guangdong province youth innovation talent project (No.2014KQNCX206) and Shaoguan city of science and technology program funded projects (No.2014CX/K233).

\section{References}

[1] Li Jin, Xie Ruhe, Liu Guanghai, et al. Establishment of cooling mathematical model and analysis of influence factors for refrigerated trucks[J]. Transactions of the Chinese Society for Agricultural Machinery, 2013,44(6): 175-182.

[2] Li Jin, Xie Ruhe, Liu Guanghai, et al. Cooling characteristics and influence parameters of multitemperature refrigerated truck[J]. Transactions of the Chinese Society for Agricultural Machinery, 2013,44(2): 128-135.

[3] Li Jin, Xie Ruhe, Liu Guanghai, et al. Research on the thermal stability of typical refrigerated trucks compartment in the outdoor sol-air temperature conditions[J]. Transactions of the Chinese Society for Agricultural Machinery, 2012,43(8): 159-166.

[4] Li Jin, Xie Ruhe. Research Progress Refrigerated Transport Equipment Technology[J]. Fluid Machinery, 2014,42(05): 82 -87.

[5] Li Jin, Xie Ruhe, Liu Guanghai. Simulation study on the compartment temperature field influence factors of perishable food refrigerated trucks[J].Food \& Machinery,2012, (3): 190-194.

[6] Li Jin, Xie Ruhe. Analysis on Temperature Fluctuations Characteristics of the Refrigerated Compartments[J]. Fluid Machinery,2011,42(01):63 -67.

[7] Long Enshen, Wang Yong, Fu Xiangzhao, et al. Experi- mental study on the temperature features inside the car parking in summer outdoors[J]. Journal of Chong qing Jianzhu University, 2003, 25(6): 49-53.

[8] Long Enshen, Wang Yong. Theoretical and experimental study on air temperature characteristics inside a car during parking and starting[J]. Journal of Chongqing Jianzhu University, 2003, 25(8): 83-88.

[9] Sun Shufeng, Wang Yiyi, Chen Liufang, et al. Simula- tion calculation on temperature dropping of the automobile air conditioner[J]. Cryogenics and super- conductivity, 2000, 28(2): 36-40.

[10] Liu Hongsheng, Chen Jiangping, Chen Zhijiu. An experimental study on the performance of mobile CO2 air conditioner[J].Automotive Engineering, 2006, 28(6):577 589.

[11] Virseda P, Pinazo J M. Heat conduction in multilayer spherical products by transfer function[J]. International Journal of Refrigeration. 1998, 21(4):285-294.

[12] Sesttrada A C, Cleland D J. Prediction of the dynamic thermal behavior of walls for refrigerated rooms using lumped and distributed parameter models[J]. International Journal of Refrigeration. 2001, 24(6):272-284. 
[13] Seem J E, Klein S A, Beckman W A, et al. Model reduction of transfer function using a dominant root method. Journal of Heat Transfer[J].1990, (11):547-552.

[14] Oakley J E, Hagan O A. Probabilistic sensitivity analysis of complex models a Bayesian approach[J].Royal statistical society,2004,66(3):751-69.

[15] TAN Bitao, CHEN Hongbin, WANG Qunshu,et al. Sensitivity analysis method on detecting ability of electro-optical system[J]. Acta Photonica Sinica, 2013,42(11):1340-1344. 


\title{
The key technology research of higher vocational education cloud platform in guizhou Based on big data
}

\author{
Yuan Linlin ${ }^{1, a^{*}}$, Qin Jin ${ }^{2, b}$,Huang Ying ${ }^{3, c}$ \\ ${ }^{1}$ Guizhou Vocational Technical Institute, Guiyang 550023,Guizhou,China \\ ${ }^{2}$ Computer Science and Technology, GuiZhou University, Guiyang 550023, Guizhou,China \\ ${ }^{3}$ Guizhou Vocational Technical Institute, Guiyang 550023,Guizhou,China \\ a120362538@qq.com, 'linlinqizhi@qq.com, 34067445@qq.com
}

Keywords: higher vocational; education cloud platform; big data

Abstract. designed and implemented a set of typical education cloud platform system, the platform adopts the double virtual network architecture, the network is divided into two layers: the underlying real device based on virtual support platform, to build the business layer.

\section{Introduction}

At present, the education informatization application has entered the transition period, is gradually by building the campus network and other infrastructure platform to deepen the application, application to drive the development of modern education transformation development. How to make use of a new generation of information technology, to realize the rapid development of education informatization education informatization construction is facing new challenges. And with the popularity of cloud computing technology and application, and promote the development of desktop virtualization, compared with the traditional PC application solutions for its low cost, high reliability, low maintenance, high security, the characteristics of flexibility, more and more popular in the education industry[1].

On the fifth plenary session of the 17 th, the state council issued "the state council on speeding up cultivating and developing strategic emerging industries decided to », put forward to develop the strategic emerging industries, the GDP ratio rose from $8 \%$ in 2015 to $15 \%$ in 2020 . A new generation of information technology as an important branch of strategic emerging industries, also got " « decision support, a reference to promote the Internet of things, cloud computing research and demonstration application.

From domestic market penetration, "twelfth five-year" period will be cloud computing industry the most critical period of growth, the cloud computing market will enter the growth stage, the industry will be rapid growth. Is expected to around 2015, cloud computing applications will begin to enter the mature stage of development. The cloud services over the next five years the average annual compound growth rate is expected to reach more than $30 \%$, the development speed is more than 6 times of traditional IT industry.

\section{DIG DATA AND CLOUD PLATFORM ADVATAGES}

Project construction from school practical teaching requirements, higher vocational education in guizhou cloud platform can have the following advantages[2]:

1) Effectively improve the quality of teaching, change the "teacher-centered" passive learning mode, for the "student-centered" mode of active learning.

Currently the vast majority of school teaching and teachers taught in the form of PPT, not according to the specific needs of each professional course provides students with the corresponding teaching scenario. After class the students no longer the unfinished homework in class learning or teaching content.

Desktop virtualization can according to the different curriculum teaching scenarios, such as database applications need Windows XP, this course teaching VFP software need, you can 
according to the specific course requirements set specific environment configuration, formed an independent teaching scene database applications.

A teacher has account and password to login his desktop whether he is at home, in office or classroom, no need to take laptop. (see the Figure1)

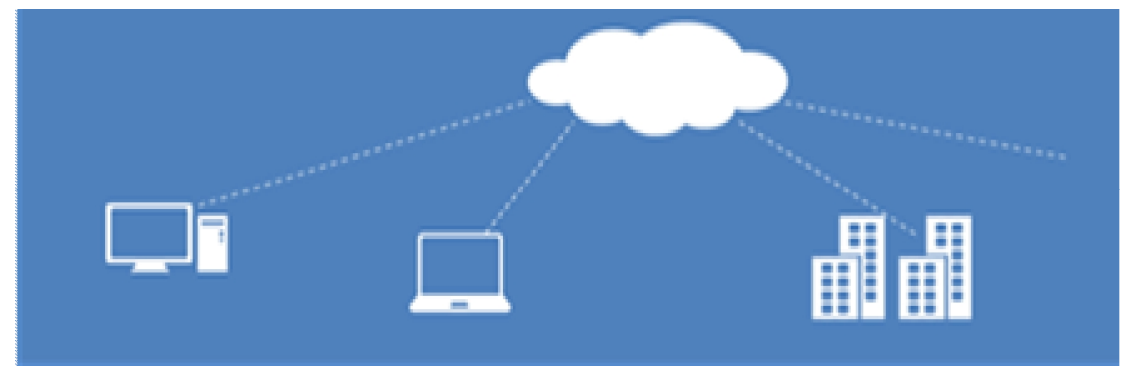

Fig .1: cloud platform advantages

According to the actual teaching situation when logging students each class can get a clean, perfect learning environment for a new desktop. Students in class can switch at any time the operating system and running environment, achieve the goal of flexible teaching. Schedule automatic import, attends class reservation function such as automatic startup for desktop virtualization and the combination of teaching, greatly reduce the work burden of administrators, improve the efficiency of teaching; Screen broadcast, lock screen, and other functions to let the teacher in class more efficient, the screen broadcast, convenient for every student can clearly see the teacher's operation and fine interpretation, lock screen function to let the students listen to the teacher only when listening to the teacher, can't surf the Internet or play games, effectively ensure the quality of multiple media teaching in the teaching.

2) Efficient unified management, reduce working intensity of management personnel

Desktop virtualization platform through the desktop computing resource pooling management, implement flexible rapid deployment, on demand. For desktop deployment and distribution, the administrator only on making image template management stage, after a batch produced and restart dozens or even hundreds of desktop, and the whole process but over ten minutes. After pooling resources management, can open desktop resources in resource utilization is low for students with special needs, time and billing, do according to the need to use, pay by using, make full use of IT resources.

Desktop virtualization as a way of cloud computing, the requirement to the terminal equipment is greatly reduced, can use a variety of computing terminal access to use desktop virtualization platform for virtual desktop. Desktop virtualization platform based on server computing architecture greatly reduce the computing needs of front-end equipment, so as to prolong the service life of the original PC terminal, with the elimination of old equipment can keep monitor continue to use, save large amount of desktop terminal input costs[3].

\section{THE KEY TECHNOLOGIES OF PLATFORM DESIGN}

1) Big data search algorithm

Once the data has been selected, the next task is to determine which of the implemented learning methods to apply. Each algorithm applies different search strategies and is applicable on only specific types of data. Beyond data selection, knowing which algorithm to use is significant challenge to making effective use of a data mining tool. In the following sections, each algorithm will be explained in terms of the data upon which it acts and examples are given for when it is applicable.

The selection of a search algorithm depends on the characteristics of the database. Applying different algorithms to the same database will lead to different discovered knowledge. A search approach can be either bottom-up or top-down. Bottom-up search uses a sequence of generalization operations to generalize the concepts; however, a top-down approach modifies the initial description by both generalization and specialization operations until the rules classify the training set correctly. 
The size of the training set determines the search strategy. The simplest strategy is an exhaustive search which applies to the entire set. However, in a real application, the search space is often large enough to make an exhaustive search inefficient. Therefore, some modified strategies were developed for improving the search efficiency. If the search strategy is represented as a tree, then the nodes are the set of objects and the edges represent operations. The initial structure is the root of the tree. The system navigates, by operations, through the search space. If there is only one operation with the best quality applied, it is a hill climber; if there are $\mathrm{n}$ best operations selected, it is a beam search where $\mathrm{n}$ is the beam width; or if all operations are fully developed, then it is called an exhaustive search. The relation among these three can be represented in the Figure 2

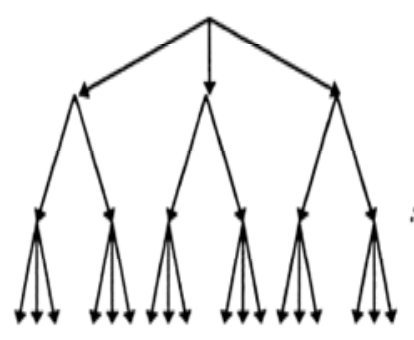

Exhaustive search

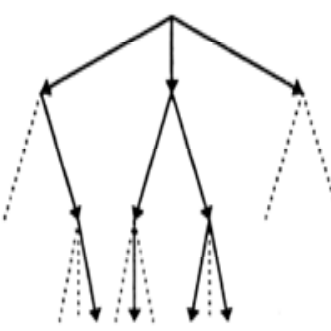

Beam search

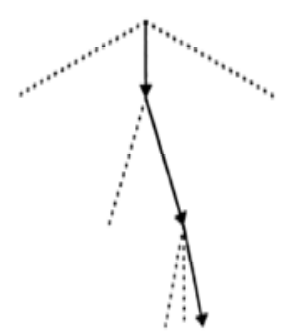

Hill climber

Fig. 2: Search strategies

Classification-rule learning involves finding rules or decision trees that partition given data into predefined classes. For any realistic problem domain of the classification-rule learning, the set of possible decision trees is too large to be searched exhaustively. In fact, the computational complexity of finding an optimal classification decision tree is NP hard. Most of the existing induction-based algorithms use Hunt's method as the basic algorithm. Here is a recursive description of Hunt's method for constructing a decision tree from a set $\mathrm{T}$ of training cases with classes denoted $\{\mathrm{C} 1, \mathrm{C} 2, \ldots, \mathrm{Ck}\}$.

Case $1 \mathrm{~T}$ contains one or more cases, all belonging to a single class $\mathrm{Cj}$ : The decision tree for $\mathrm{T}$ is a leaf identifying class $\mathrm{Cj}$.

Case $2 \mathrm{~T}$ contains no cases: The decision tree for $\mathrm{T}$ is a leaf, but the class to be associated with the leaf must be determined from information other than $\mathrm{T}$.

Case $3 \mathrm{~T}$ contains cases that belong to a mixture of classes: A test is chosen, based on a single attribute, that has one or more mutually exclusive outcomes $\{\mathrm{O} 1, \mathrm{O} 2, . ., \mathrm{On}\}$. $\mathrm{T}$ is partitioned into subsets $\mathrm{T} 1, \mathrm{~T} 2, \ldots, \mathrm{Tn}$, where Ti contains all the cases in $\mathrm{T}$ that have outcome Oi of the chosen test. The decision tree for $\mathrm{T}$ consists of a decision node identifying the test, and one branch for each possible outcome. The same tree building machinery is applied recursively to each subset of training cases.

2) education cloud platform stucture

Virtualization platform is a distributed computing platform, overall architecture as shown(see the Figure3): 


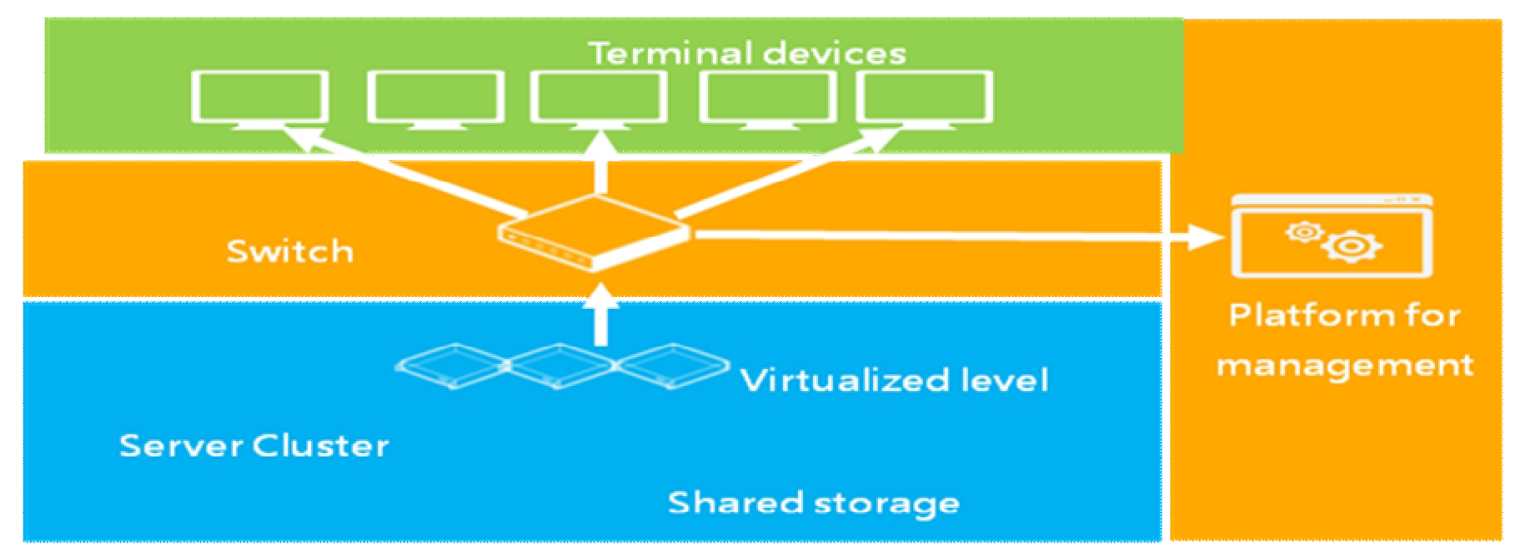

Fig.3: cloud platform stucture

Cloud computing is the future development trend of education informatization platform, while education cloud with special requirements according to their aptitude, universal cloud platform is not very suitable. In view of the personalized needs in the field of education, the author participated in the team's education cloud platform design and implementation. Platform system adopts double virtual network architecture, in terms of scalability, flexibility, safety, high efficiency advantages, have education cloud need all kinds of big data processing capabilities.

\section{SUMMARY}

Education cloud platform research and development is a long-term work, there are still a lot of future application research and development as well as the core technology research and development work to do. Overall architecture design, the cloud platform has been done of virtualization, cloud computing resources and resource overdraw function, the future will also further deepen resources use way and architecture, trying to maximize resource utilization, using the risk minimization. Can draw lessons from the financial credit in the field of risk assessment and control model, the cloud computing platform to establish similar financial resources reserve mechanism of the deposit reserve system.

\section{Fund Project Sponsors}

This paper is supported by the Commissioned by the national Open University, number :G14A3602W; This paper is supported by the Guizhou institute of vocational and technical college vocational education subject, number : 15GH04; and The National Natural Science Fund;NO:61562009, and The National Natural Science Fund;NO:61562009; and the Guizhou University to introduce talents research project NO:[2012]028

\section{References}

[1] Jian-cheng QIN,Zhong-ying BAI. Design of new format for mass data compression[J]. The Journal of China Universities of Posts and Telecommunications . 2011 (1)

[2] Michael Armbrust,Armando Fox,Rean Griffith,Anthony D. Joseph,Randy Katz,Andy Konwinski,Gunho Lee,David Patterson,Ariel Rabkin,Ion Stoica,Matei Zaharia. A view of cloud computing[J]. Communications of the ACM . 2010 (4)

[3] Min Long,Fei Peng,Shuisheng Qiu,Yanfeng Chen. Implementation of a new chaotic encryption system and synchronization 11 This project was supported by the National Natural Science Foundation of China (60372004), and the Natural Science Foundation of Guangdong Province (31445, 20820).[J]. Journal of Systems Engineering and Electronics . 2006 (1) 